\section{Forget me not - the role of the general dental practitioner in dementia awareness}

\author{
G. McNamara, ${ }^{1}$ J. Millwood, ${ }^{* 2}$ Y. M. Rooney ${ }^{3}$ and K. Bennett ${ }^{4}$
}

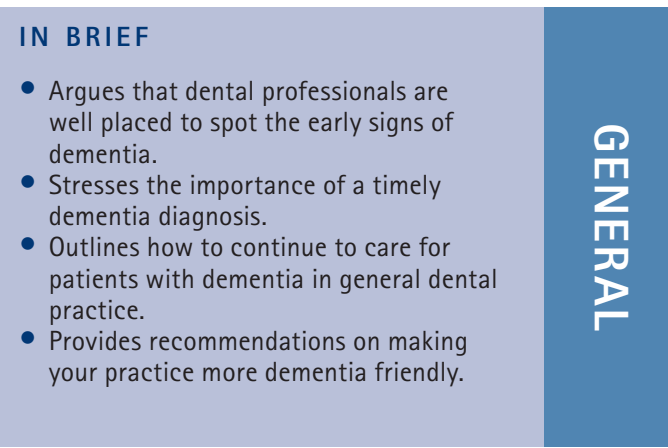

This paper examines the role of the general dental practitioner (GDP) in dementia awareness, using the close patient-
practitioner relationship to spot the early signs and plan for future oral healthcare. Each and every member of the dental
team plays an important role in the patient journey through their dental visit and helps address the barriers they face.
The significance of promoting dementia friendly environments cannot be undervalued and the GDP has a key role in
maintaining their patients' quality of life.

\section{ARE GENERAL DENTAL PRACTITIONERS THE LAST BASTION OF CONTINUING CARE?}

The changing role and responsibilities of general practitioners (GPs) mean that the 'family doctor', with whom you have a personal relationship with, is too often a dying concept - particularly in large urban areas. In some areas this has been replaced by a sense of you get what you're given, 10 minute appointments (and double appointments if you want to discuss more than one health complaint) and frantic calling first thing in the morning if you want to see a GP on the day.

But what about the family dentist? Does this concept remain alive and well? I would argue it does. Many dentists have a personal relationship with their patients; know what they do for a living, whether they have children and where they are going on holiday. They are more likely to treat the whole family and so are able to build up a comprehensive picture of their patients as an individual. So it follows that they may

${ }^{1}$ Head of Policy and Public Affairs, Alzheimer's Society, Devon House, 58 St Katherine's Way, London, E1W 1LB; ${ }^{2}$ Specialist in Special Care Dentistry, Committee member of the British Society of Gerodontology, Derbyshire Community Health Services, Dental Clinic Loughborough Hospital, Epinal Way, Loughborough, LE11 5JY; ${ }^{3}$ Speciality Registrar Special Care Dentistry, King's College NHS Foundation Trust, Department of Community Special Care Dentistry, C/0 161 Denmark Hill, Camberwell, London, SE5 8EF; ${ }^{4}$ Press Officer, Alzheimer's Society, Devon House, 58 St Katherine's Way, London, E1W 1LB

*Correspondence to: Joanna Millwood

Email: joanna.millwood@ntlworld.com

\section{Refereed Paper}

Accepted 22 July 2014

DOI: 10.1038/sj.bdj.2014.760

${ }^{\circledR}$ British Dental Journal 2014; 217: 245-248 be more likely to spot if their patient doesn't quite seem themselves and notice subtle changes in behaviour.

So, what if you noticed that a regular patient, someone you have treated for years, was having trouble with their memory? The last time you saw them they had trouble recalling their granddaughter's name. Today, they have forgotten whether they brushed their teeth this morning and you have noticed a general decline in their oral hygiene. You know they suffered a ministoke about 12 months ago, but this is quite out of character. In the years you have known them they have also been impeccably presented and organised and yet this no longer seems to be the case. You have got a nagging feeling that something is wrong - but would you choose to say something?

\section{DEMENTIA - \\ A NATIONAL CHALLENGE}

We have heard it heralded as a 'timebomb', 'epidemic', and 'our greatest health challenge yet'. There can be no doubt that in recent years dementia has rocketed up the public agenda. With an ageing population and an annual cost of $£ 23$ billion to the UK economy, the condition is finally being accepted as a national challenge by Government with the Prime Minister launching his own challenge on dementia. With 800,000 people living with dementia in the UK, the truth is dementia will soon touch every family in the land and yet despite the welcome focus from the Government we still have some way to go until society fully understands the realities of the condition and the complexities of how dementia affects each individual differently. Too frequently people hear the word dementia and picture an older person in residential care, unable to live well. Yet this is not the full story and if you spend an afternoon at an Alzheimer's Society service (for example, a Singing for the Brain group or Dementia café) you will see that dementia and despair do not have to go hand-in-hand. Nor is it exclusively 'an older person's disease' as there is an estimated 17,000 people under the age of 65 living with the condition in the UK. The author Terry Pratchett, who was diagnosed with a rare form of dementia, posterior cortical atrophy, at the age of 59, has done a great deal to raise awareness of young-onset dementia. However, the reality is that as many as one in three people over the age of 65 will die with dementia but, despite its prevalence, the condition remains vastly under-diagnosed - with fewer than half of people $(48 \%)$ who are living with the symptoms in England receiving a formal diagnosis. ${ }^{1}$

'I knew that something was wrong. Things at home weren't quite right. I was losing things and stopped being able to recognise things. One day I didn't recognise a glass. I felt depressed and demoralised, took on too much at work and got sacked.'

'At first, the symptoms were put down to depression, but when I was told "You've got Alzheimer's disease", I cried with relief. After 18 months of trying to remember if I'd read the paper, I finally realised what was really happening.' (Person with dementia)

\section{THE IMPORTANCE OF RECEIVING A TIMELY DEMENTIA DIAGNOSIS}

Historic experience of people with dementia, their families and carers articulates just how important getting a timely diagnosis 
of dementia is. A diagnosis enables access to treatments and support; acting as a catalyst for discussions about how to live with dementia including power of attorney (for finance and health and wellbeing), maintaining health, driving and advanced care planning while the person can still actively participate. Crucially, it gives people valuable time when the short and long-term future can be discussed before the situation becomes critical. There are numerous barriers to a dementia diagnosis, which are steadily being broken down, but some of the more persistent is the stigma attached to dementia and the misplaced perception that it is a natural part of ageing that you can do little to nothing about. In addition, as there is no cure for dementia and only a few treatments for Alzheimer's disease (the most common form of dementia) with limited effectiveness, some clinicians question the purpose of a diagnosis. Those of this opinion are ignoring the importance support and information can play in helping people to feel more in control. However, the tide is turning with the success of public awareness campaigns and media interest in dementia leaving people more informed and willing to talk about the condition and the symptoms of memory loss. Coupled with this, in 2013 the GP contract was revised to tackle the low levels of dementia diagnosis rates with the introduction of the dementia Directed Enhanced Service (DES). The DES promotes a case finding approach based on a great deal of what we know about comorbidities of dementia, namely that those with vascular risk factors are at much greater risk - in some cases estimated to be over 50\% - of dementia. This has resulted in clinicians asking those who are at a higher risk of developing dementia if they are worried about their memory in order to refer them for appropriate tests. A question about dementia and the signs and symptoms has also been added to the NHS Health Check. A survey of 382 GPs in England, Wales and Northern Ireland found that $88 \%$ of clinicians agree that there are benefits to an early diagnosis of dementia. ${ }^{2}$ However, despite this, the DES has been regarded as controversial by some GPs, with concerns that the policy of incentivising could lead to over diagnosis. The DES has also erroneously been labelled as population screening by some. Population screening for dementia does not exist in the UK and there are no plans to introduce it. The rationale behind the scheme is that by equipping clinicians with the training and tools needed to understand those most at risk of developing dementia, and putting them in the driving seat of any clinical assessment, we protect individuals against inappropriate interventions. It is not designed to 'force' a diagnosis on people and absolute respect must be given to anyone not wishing to know. Most people with dementia will live for years with the condition and there is much that can be done to help them along their journey and enable them to live well. Earlier diagnosis will not alter the ultimate outcome, but the better informed and prepared people are, the better equipped they will be to tackle the challenges that dementia presents.

'I was worried about my husband, Al, for a couple of years before he got a diagnosis - but getting a diagnosis was the best thing that could have happened to us. Al remained very capable, but sometimes struggled with little but important things like forgetting he'd promised to pick me up from the hairdressers or getting in a muddle when it came to banking. He was put on Aricept and I'm convinced it gave him a new lease of life. We even went on to win medals for our dancing because Al was able to take part in private dancing lessons in Newcastle.

We were very lucky because we had an excellent dentist in Amble. He was very good with Al; very kind and understanding, which was extremely important because as well as dementia Al had a partly paralysed throat condition. Having a dentist who is aware of the problem means a person with dementia can be treated with the dignity and patience that are vital. To be brusque with someone who has dementia will cause problems. It is vital dentists are aware of how dementia can progress so they can communicate in an appropriate way, so their patient can understand and feel safe and secure and can trust their dentist.' (Barbara Dow, former carer of a person with dementia).

\section{SPOTTING THE SIGNS AND SYMPTOMS OF DEMENTIA IN THE DENTAL CHAIR: THE ROLE OF THE GDP AND DENTAL TEAM IN PROVIDING HOLISTIC, PERSON-CENTRED CARE}

Continuity of care is a valuable aspect of general dental practice. Often patients, and their family members, attend over a long period of time and a beneficial rapport can be established. Even with patients who are anxious or reluctant the dentist can build a professional relationship and understanding. As well as the clinical and technical aspects of dental care, the dental team play a vital role in presenting a patient-centred approach to each dental appointment. The practice receptionist and dental nurse may establish a good relationship with the patient and family members, observing any subtle dynamics or support given. A holistically minded general dental practitioner (GDP) and dental team are well positioned to spot any changes in memory, behaviour and ability to communicate, which may be indicative of the early stages of dementia. While not suggesting that a diagnosis can, or should be, made in the dental chair, having a regular and open dialogue with a patient and, where appropriate, their family could provide the context for discussion concerns about their memory. The dentist can signpost a patient to their GP or resources such as Alzheimer's Society's Worried about your memory? ? $^{3}$

\section{WHAT TO LOOK OUT FOR: THE SIGNS AND SYMPTOMS OF DEMENTIA}

Every person is unique and will experience dementia in their own way. The different types of dementia tend to affect people differently, especially in the early stages. However, the most common signs and symptoms of dementia include:

- Day-to-day memory - difficulty recalling recent events or remembering what was planned for the visit or said at the last appointment

- Language - difficulties recalling names of people or objects, following a conversation or finding the right word for something

- Visuospatial skills - problems judging distances (for example, on stairs) and seeing objects in three dimensions

- Orientation - losing track of the day or date, or becoming confused about where they are

- Problems with thinking and reasoning

- Concentrating, planning or organising difficulties grasping treatment planning suggestions or anxious about options given.

As well as these cognitive symptoms, a person with dementia will often have changes in their mood. For example, they may become frustrated or irritable, withdrawn, anxious, easily upset or unusually sad.

\section{HOW TO RAISE CONCERNS: APPROACHING THE SUBJECT OF DEMENTIA}

The individual personality of a dentist and the relationship with the patient dictate the communication style used in approaching this subject. For many it may be a sensitive and tactful approach with gentle probing and leading questions to investigate difficulty with memory or changes in behaviour. For others it may be a direct, clinical approach with frank, objective questions such as: 'Have you noticed any change in your memory?'; 'Do you struggle to recall things you have heard, seen or read?'; 'Do you ever forget names of people or everyday objects?'; 'Do you have any difficulty in 
following conversations?' Confidentiality is central to trust-based relationship between clinicians and patients, and appropriate information sharing is vital for the provision of safe and effective care. General Dental Council (GDC) Standards for the dental team ${ }^{4}$ stipulate that dentists should ensure they explain to the patient the circumstances in which we may share information, that we seek the patient's consent to do so wherever possible and that in information sharing we are prepared to justify those decisions and any action taken. If a dentist feels that there may be some evidence of memory difficulty or deterioration they can mention to the patient that they wish to write to the GP suggesting the need for a more thorough check up or review. The dentist can write briefly to the GP, describing their observation and the reason for concern, requesting that the patient be reviewed. If there is concern regarding the patient's mental capacity, advice or confirmation can be requested.

\section{HOW TO CONTINUE TO CARE FOR PATIENTS WITH DEMENTIA IN GENERAL DENTAL PRACTICE}

\section{Oral health and dementia}

It is well recognised that oral health is likely to decline in the later stages of dementia. Oral hygiene may be compromised, with an increase in periodontal disease, higher levels of decay (both coronal and cervical) and difficulty wearing dentures. Poor oral care and an increase in oral disease can cause difficulty with eating and pain or discomfort; this can precipitate behavioural changes for patients who are no longer able to effectively communicate their problems. ${ }^{5}$

For comprehensive guidance on oral healthcare for people with dementia refer to British Society of Gerodontology (BSG) guidelines available free online through the BSG website (http://www.gerodontology. com). ${ }^{6}$

\section{Assessment for dental treatment and treatment planning}

Once a person has a dementia diagnosis, it is important to establish an individual, longterm dental care plan. The long-term objective is to eliminate pain, control infection and prevent new disease. This is facilitated by a timely dementia diagnosis as the person in the early stages of the condition is more likely to be receptive to treatment and able to take an active role in decision making.

Treatment planning must take account of the stage of the illness and the level of cognitive impairment. Ideally a dentist should be part of the multidisciplinary care team for people with progressive neurogenic disorder from diagnosis, so that oral care can be planned throughout the disease process and does not become crisis management in the later stage of dementia.

From the outset, it is important to work in partnership with the carer and/or family to obtain an accurate assessment of cognitive impairment. They should also be able to advise how well the person is maintaining a dental hygiene routine, whether they remain physically able to brush their teeth and whether they may benefit from an electric toothbrush or a brush with an adapted handle.

The dental care plan must take into account that as dementia progresses, the person affected may become less able to:

- Express their needs or wishes and to explain what they want

- Understand and explain dental symptoms such as pain

- Take part in the decision making process about treatment

- Give informed consent

- Tolerate dental interventions

- Perform daily living activities such as oral hygiene

- Understand that oral hygiene needs to be carried out.

In the early stages of dementia most patients will be best cared for in general dental practice. Good restorative and rehabilitative care is usually possible, key teeth can be identified (for example, canines, molars, occluding pairs) and restored. Restorative treatment should be high quality and low maintenance. Advanced restorative treatment should only be planned in the knowledge that, should the individual no longer be able to provide oral self-care, a care-giver is prepared to take on this role. Rigorous preventive measures (both homeand surgery-based) should be put in place at this stage so that they become routine for both the individual and their carer. Prescription of high fluoride toothpaste may be considered and professional application of high fluoride varnish twice yearly may be beneficial. ${ }^{7}$ In the middle stages of dementia the focus of oral care changes from restorative and rehabilitative to maintenance and prevention. During this stage the person is often relatively physically healthy but increasingly cognitively impaired. Patients with support may still be able to receive dental care with little or no difficulty. For some, however, changes in behaviour including agitation or aggression may occur when a person feels threatened, intimidated or agitated. With reduced powers of logic and reason and heightened emotional responses, people may lash out where they previously might have behaved in a very different way. At this point it may be decided that the patient no longer has the capacity to consent to their dental treatment. This is where the Mental Capacity Act states that we must assess capacity, act in the patient's best interests and choose the least restrictive treatment option. Having already been involved in the treatment decisions a patient has made previously, this is where the GDP is truly valuable, especially so as they are already aware of the people who may wish to be kept in communication about the patient's care. At this stage the GDP may also choose to refer to a special care dental service for specialist advice and continuing care that may include sedation or general anaesthesia for treatment. The decision will be based on the individual's ability to cooperate, dental treatment needs, general health and social support. Rigorous prevention should be continued and more frequent recall visits and support for carers employed, as appropriate. The role of dental care professionals in helping to maintain good oral care is recommended. In the later stages of dementia some patients are still cared for by their GDP. Most people will become increasingly frail due to the progression of the illness. Treatment at this stage focuses on prevention, maintaining oral comfort and palliative emergency treatment. Dental interventions should be kept as non-invasive as possible for example, using Carisolv for caries removal, atraumatic restorative techniques (ART) such as glass ionomer cement restorations, and regular application of high fluoride varnish to control root caries.

As dementia progresses it can become increasingly disruptive for patients to attend visits outside of their normal environment; in this situation domiciliary dental care may be best provided. A referral may be made to local domiciliary services. The referral should provide effective transition of care to the next dental team and as such should include information on medical practitioners, family members/carers and a brief dental history with any radiographs, as these will not be possible in a domiciliary setting.

In Evidence-Based Dentistry, Carson and Edwards ${ }^{8}$ describe the most common barriers to delivering oral care to older people in care homes as lack of adequate equipment and facilities, poor financial reimbursement, lack of training and loss of time from private practice. Use of treatment modalities such as a combination of oral or intranasal sedation with intravenous sedation or general anaesthesia may be considered but the benefit from treatment has to be weighed up and at least balanced with the risks and difficulties of providing it in terms of cooperation, consent, medical status etc. The following British Society of Gerontology guidelines are useful when considering strategic, long-term, oral care 
planning for people with dementia:

- Instigate appropriate preventive measures to minimise dental disease as soon as possible

- Undertake dental intervention in the early stages of the condition to manage outstanding dental treatment needs

- Ensure dentures are named, cleaned professionally on a regular basis and renewed using a duplication technique when their replacement is necessary

- Instigate regular review tailored to the individual's needs to maintain the oral status quo, avoid pain and minimise further interventions

- Ensure that fear, stress and embarrassment for the carer and the person with dementia are minimised by adopting an 'open-door' approach and providing back-up and support.

\section{Tolerating oral and/or dental interventions}

Compliance for oral healthcare procedures and dental care does not necessarily decline with advancing dementia. Even people with very advanced dementia can retain the capability of tooth brushing if handed a toothbrush.

The ability to comply with oral hygiene procedures and dental care is often influenced by past dental behaviour and experiences. ${ }^{9}$ Some people with dementia who have had regular dental treatment throughout their lives seem to remember what they are expected to do in the dental surgery. They have little difficulty cooperating with simple procedures until the late stage of the disease. Familiar surroundings, routines and people may be reassuring and aid cooperation.

\section{ENSURING DENTAL PRACTICES WORK TOWARDS BECOMING DEMENTIA FRIENDLY}

In pursuit of making your practice dementia friendly in its ethos, the awareness, training and communication of the dental team are key - coupled with practical steps to support appointments and giving information. The following are some important considerations when treating people with dementia:

- Recognise that some people have good days and bad days. If possible, dental care is better postponed to a good day and to the individual's best time of day

- Some people with dementia have short attention spans, meaning their ability to cooperate is decreased. Dental appointments should be kept within the individual's capacity to cope

- Sending out appointment reminders. This is already common place in a number of practices; however, flexibility in the use of media such as text messages or emails may be required. Patients may appreciate a telephone call the day before their appointment as a simple reminder.

- While dementia friendly architecture and design may be unrealistic, simple, thoughtful improvements can be made to the environment of the practice to increase the accessibility to older patients in general, and to patients living with dementia:

1. Noise reduction may support inclusion and reduce confusion. A person with dementia may be disorientated and have difficulty with loss of balance as a result of trying to orientate themselves in environment that is noisy and over stimulating

2. Good lighting is particularly important, making the best of natural daylight, increasing light levels, minimising glare, avoiding sudden changes in light contrast and good colour definition can be beneficial

3. Flooring should be matt and an even colour if possible

4. Signs and names should be easy to read, consider large font sizes or easy read format. Ensure seating of an appropriate height which allows ease into a standing position, provides good support and consider the layout of seating in the waiting room

5. Patients may appreciate age appropriate magazines.

Additionally, all members of the dental team could consider becoming a 'dementia friend'. Dementia Friends is a national initiative run by Alzheimer's Society. It aims to change the way the nation thinks, talks and acts when it comes to dementia. The campaign hopes to create a more dementia-friendly society by encouraging one million people to become dementia friends. Dementia friends learn more about what it is like to live with dementia and how to turn that understanding into action - anyone of any age can become a dementia friend by attending a $45 \mathrm{~min}$ faceto-face awareness session run by a dementia champion (a volunteer who has undergone a 1 day training session). It is easy to find a local session on the Dementia Friends website.

With an emphasis on 'improving the lives of people with dementia' in political and public debate, and with a healthcare service stretched to capacity, the question arises of who will take responsibility for supporting those with dementia. When dementia is portrayed as a 'national crisis' and 'burden', the problem seems too large in scale to conquer. However when broken down, ultimately the challenge is for us all to take small steps and actions within our existing lives and jobs to be more aware of dementia and offer a helping hand to those affected. In dentistry, that includes awareness of signs and symptoms, encouraging open conversations with patients and knowing what information you can signpost people to if they're worried and who can help them get a diagnosis.

Each and every member of the dental team plays an important role in the patient journey through their dental visit. Being aware of the difficulties some patients face in booking and keeping their appointments, accessing the dental surgery and even getting as far as the dental chair is the first step to addressing the barriers they face. It also worth remembering that a patient with dementia will have more of the same anxieties about dental care as the rest of the population. As a profession accepted as a routine 6-12 monthly part of a patient's life, you are ideally placed to openly promote dementia friendly environments and play an important role in maintaining your patient's quality of life.

This article draws on the Guidelines for the development of local standards of oral health care for people with dementia, a policy document prepared by Janice Fiske, Heather Frenkel, Janet Griffiths and Vicki Jones on behalf of the British Society of Gerodontology and the British Society for Disability and Oral Health; funded by the Department of Health, published in Gerodontology, volume 23, supplement 1, December 2006. The authors would like to offer thanks to the British Society of Gerodontology. ${ }^{10}$

1. Department of Health. Dementia: a state of the nation report on dementia care and support in England. London: DH, 2013.

2. Alzheimer's Society. Worried about your memory? GP survey. 2012. Report available online at http:// www.alzheimers.org.uk/site/scripts/download_info. php?fileID=1476 (accessed September 2014).

3. Alzheimer's Society. Worried about your memory? London: Alzheimer's Society, 2013. Online booklet available at http://www.alzheimers.org.uk/site/ scripts/documents_info.php?documentID=1138 (accessed July 2014).

4. General Dental Council. Standards for the dental team. London: GDC, 2013.

5. Cohen-Mansfield J, Lipson S. The underdetection of pain of dental aetiology in persons with dementia. Am J Alzheimers Dis Other Demen 2002;17: 249-253.

6. Fiske J, Frenkel H, Griffiths J, Jones V. Guidelines for development of local standards of oral health for people with dementia. Gerodontology 2006; 23: 3-32.

7. Public Health England. Delivering better oral health: an evidence based tool kit for prevention. 3rd ed. London: Department of Health, 2014. Online guidelines available at https://www.gov.uk/ government/uploads/system/uploads/attachment data/file/319471/DBOHv3JUNE2014.pdf (accessed July 2014).

8. Carson S J and Edwards M. Barriers to providing dental care for older people. Evid Based Dent 2014; 15: 14-15.

9. Hilton C, Simons B. Dental surgery attendance among patients with moderately advanced dementia attending a day unit: a survey of carer's views. Br Dent J 2003; 195: 39-40.

10. Alzheimer's Society. Dental care and dementia Factsheet 448. London: Alzheimer's Society, 2013. Online information available at http://www. alzheimers.org.uk/factsheets (accessed July 2014). 